\title{
Coulomb self-energy integral of a uniformly charged d-cube: a physically-based method for approximating multiple integrals
}

\author{
Josep Batle ${ }^{1}$, Orion Ciftja ${ }^{2}$, Mosayeb Naseri ${ }^{3}$, Mahmood Ghoranneviss ${ }^{4}$, Koji Nagata ${ }^{5}$ and Tadao Nakamura ${ }^{6 *}$ \\ ${ }^{1}$ Departament de Física, Universitat de les Illes Balears, \\ 07122 Palma de Mallorca, Balearic Islands, Spain \\ ${ }^{2}$ Department of Physics, Prairie View A\&M University, \\ Prairie View, TX 77446, USA \\ ${ }^{3}$ Department of Physics, Kermanshah Branch, \\ Islamic Azad University, Kermanshah, Iran \\ ${ }^{4}$ Plasma Physics Research Center, \\ Science and Research Branch, \\ Islamic Azad University, Tehran, Iran \\ ${ }^{5}$ Department of Physics, \\ Korea Advanced Institute of Science and Technology, \\ Daejeon 305-701, South Korea \\ ${ }^{6}$ Department of Information and Computer Science, \\ Keio University, Kohoku-ku, 3-14-1 Hiyoshi, \\ Yokohama, Kanagawa 223-8522, Japan
}

(Dated: December 8, 2016)

\begin{abstract}
We report a concise expression for the electrostatic Coulomb self-energy of a uniformly charged cube with arbitrary dimensionality. The result, conveniently expressed in integral form, shows the unique behavior of the system as dimensionality increases. We use the case study of a uniformly charged square and cube to illustrate the effectiveness of the method employed. The resulting integral expression of the Coulomb self-energy of a multi-dimensional cube can be calculated numerically at very high accuracy. Numerically exact values of Coulomb self-energy obtained this way provide an excellent tool to gauge the validity of various computational techniques including a straightforward discretization method employed in this work. The proposed method relates the self-energy multi-variable integral to energy contributions coming from a finite discrete system of point charges with the understanding that the number of point charges gradually increases towards the continuum limit. We achieve very good accuracy by using an expression with only two terms where the second term increases considerably the accuracy of computation of continuum integrals and represents a systematic first-order correction to the finite energy of the discrete system. The applied numerical method turns out to be very reliable as shown by the results obtained for cubic domains with dimensionality varying from two to five. The method can be easily generalized to other types of domains with arbitrary geometry.
\end{abstract}

PACS numbers: 45; 41.20.Cv; 45.50.Jf

Keywords: Electrostatic potential, Coulomb interaction, Uniformly charged $d$-cube, Physics-based numerical method.

\section{INTRODUCTION}

Prediction of crystallization of electrons by Wigner [1] has influenced many branches of science. Realization of corresponding experimental settings has led to the observation of Wigner crystals in various systems such as superfluid Helium [2] or GaAs/GaAlAs quantum wells [3]. Wigner crystals in one dimension (1D) have been produced in carbon nanotubes [4], as well. Recent experimental advances in nanoscience and nanotechnology [5] have boosted the theoretical interest on the properties

* E-mail address (JB): jbv276@uib.es of finite systems (typically, electrons interacting via a Coulomb interaction potential). Research closely related to the original Wigner problem has triggered a diverse body of literature such as the one focused on the properties of ionic Coulomb crystals. In particular, there has been renewed interest on the so-called Thomson problem $[6,7]$, the search of the minimal energy state of $N$ point charges confined to the surface of a sphere. The Thomson problem plays a central role in many fields and is well studied in the literature [8-11]. The arrangement of charged particles in two-dimensional (2D) systems has also been studied [12-15].

Often, the interaction of a large number of charges is treated in the continuum. For such a situation, one faces the difficult task of calculating the Coulomb electrostatic energy of interaction between charge distributions spread over arbitrary three-dimensional (3D) domains. The simplest scenario is that of a uniform charge distribution. However, the calculations are not straighforward even in this case and depend on the geometry of the charge dis- 
tribution under consideration. For instance, calculation of the resulting Coulomb integrals corresponding to the self-energy of a uniformly charged square or cube turns out to be a highly non-trivial task. These type of integrals are often found in physics and chemistry theoretical models [16-19], and are computationally costly. Their numerical evaluation encounters two main drawbacks. On the one hand, these integrals are multi-dimensional, thus they require a large number of cubature points. On the other hand, the integrand function has a singularity whenever the coordinates of two points $P$ and $P^{\prime}$ coincide. This leads to slow convergence in standard numerical integration methods.

In two recent contributions, Ciftja [20,21] was able to elegantly avoid the painstaking task of multiple integration in obtaining the Coulomb self-energy for a uniformly charged square and cube. In the present work, we shall extend his method to calculate the Coulomb self-energy of a uniformly charged cube with an arbitrary dimensionality, a $d$-cube. Obviously, the calculation of the Coulomb self-energy for arbitrary charge distributions (those without cubic, circular, etc. symmetry) cannot be handled analytically. For example, the calculation of the Coulomb energy of a 3D distribution of charges represented by six-dimensional integrals requires use of specific computational techniques that are not easy to implement [22].

In order to overcome some of these computational challenges, we propose an easy to implement discretization method for the calculation of the self-energy of a uniform charge distribution. In this method, the self-energy of a uniform charge distribution is approximated as a sum of only two terms. The first term is the mere total energy of a discrete system of point charges placed on a lattice. The discrete system mirrors the features of the uniform charge distribution that is emulating (same total charge, same overall volume/geometry, etc.). The nature of the lattice chosen to set up the array of point charges (cubic, hexagonal, etc) is optional. The second term represents a first-order energy correction that scales in a well-known fashion as a function of the number of point charges of the discrete system. We gauge the accuracy of the approach by comparing the results to numerically exact values obtained for a $d$-cube. The good agreement observed suggests that this approach may work well for other uniform charge distributions with a different geometry.

The remainder of this article is structured as follows: In Section II, we revisit the approach that leads to the exact calculation of the Coulomb self-energy of a uniformly charged square $(d=2)$ and cube $(d=3)$. Section III extends the previous results to a uniformly charged $d$ cube with an arbitrary dimensionality. In the process, we report an interesting result for the dependence of the Coulomb self-energy of a uniformly charged $d$-cube as a function of its dimensionality. The discretization approach for the calculation of integrals where dimensions up to five are considered (ten-fold integration) is unfolded in Section IV. Conclusions are drawn in Section V.

\section{TOTAL (CONTINUUM) COULOMB SELF-ENERGY OF A UNIFORMLY CHARGED SQUARE AND CUBE}

Let us consider a 2D square domain of length $L$ given by $D:[0, L] \times[0, L]$ in a Cartesian system of coordinates. From now on we work with a Cartesian system coordinates. It is assumed that this domain contains a total amount of charge, $Q$ which is uniformly spread. Hence, the uniform surface charge density in this case is: $\rho(\vec{r})=\rho_{0}=Q / L^{2}$. The total electrostatic energy due to the Coulomb interaction between all infinitesimal charges inside the square, namely, the electrostatic Coulomb selfenergy of the body is:

$$
\begin{gathered}
U=\frac{k}{2} \int_{D} d \vec{r} \int_{D} d \overrightarrow{r^{\prime}} \frac{\rho(\vec{r}) \rho\left(\overrightarrow{r^{\prime}}\right)}{\left|\vec{r}-\overrightarrow{r^{\prime}}\right|}=\frac{k \rho_{0}^{2}}{2} \int_{D} d \vec{r} \int_{D} d \overrightarrow{r^{\prime}} \frac{1}{\left|\vec{r}-\overrightarrow{r^{\prime}}\right|} \\
=\frac{k \rho_{0}^{2}}{2} \int_{0}^{L} d x \int_{0}^{L} d y \int_{0}^{L} d x^{\prime} \int_{0}^{L} d y^{\prime} \frac{1}{\sqrt{\left(x-x^{\prime}\right)^{2}+\left(y-y^{\prime}\right)^{2}}}
\end{gathered}
$$

where $k$ is Coulomb's electric constant, $\vec{r}\left(\vec{r}^{\prime}\right)$ are 2D position vectors and $d \vec{r}\left(d \overrightarrow{r^{\prime}}\right)$ are area elements. Since all Cartesian coordinates in Eq.(1) can be rescaled in units of $L$ (and we shall continue using the same dummy letters), the ensuing problem is to compute the integral:

$$
\frac{2 U}{k \rho_{0}^{2} L^{3}}=\int_{0}^{1} d x \int_{0}^{1} d y \int_{0}^{1} d x^{\prime} \int_{0}^{1} d y^{\prime} \frac{1}{\sqrt{\left(x-x^{\prime}\right)^{2}+\left(y-y^{\prime}\right)^{2}}} .
$$

For the given uniform surface charge density in 2D, we have:

$$
k \rho_{0}^{2} L^{3}=\frac{k Q^{2}}{L} \quad(2 D)
$$

a quantity that represents the expected unit of energy. Although the calculation of the integral in Eq.(2) is not trivial, it can be carried out analytically using very lengthy direct integration techniques. The integral in Eq.(2) can be computed in a different way [20,21], by taking advantage of the algebraic form of Coulomb's interaction potential and using the following useful identity:

$$
\int_{0}^{\infty} d x e^{-a x^{2}}=\frac{\sqrt{\pi}}{2 \sqrt{a}}
$$

where $a$ is a real and positive parameter. The remarkable utility of this identity lies in the fact that the output has the desired functional form for the electrostatic energy. Hence, we can write:

$$
\frac{1}{\sqrt{\left(x-x^{\prime}\right)^{2}+\left(y-y^{\prime}\right)^{2}}}=\frac{2}{\sqrt{\pi}} \int_{0}^{\infty} d u e^{-u^{2}\left[\left(x-x^{\prime}\right)^{2}+\left(y-y^{\prime}\right)^{2}\right]} .
$$


Taking into account the symmetries in Eq.(5), the final result for the Coulomb self-energy can be cast as:

$$
\frac{U}{k \rho_{0}^{2} L^{3}}=\frac{1}{\sqrt{\pi}} \int_{0}^{\infty} d t f(t)^{2}
$$

where the auxiliary function $f(t)$ is given by:

$$
f(t)=\sqrt{\pi} \frac{\operatorname{erf}(t)}{t}+\frac{e^{-t^{2}}-1}{t^{2}}
$$

and $\operatorname{erf}(x)=\frac{2}{\sqrt{\pi}} \int_{0}^{x} d z e^{-z^{2}}$ is an error function. Note that $U /\left(k \rho_{0}^{2} L^{3}\right)$ represents the value of the Coulomb self-energy of a uniformly charged $2 \mathrm{D}$ square in units of $k Q^{2} / L$ as seen from Eq.(3).

One can calculate the integral, $\int_{0}^{\infty} d t[f(t)]^{2}$ in Eq. (6) by first writing: $f(t)=f_{1}(t)+f_{2}(t)$ where $f_{1}(t)=$ $\sqrt{\pi} \operatorname{erf}(t) / t$ and $f_{2}(t)=\left(e^{-t^{2}}-1\right) / t^{2}$. After completing the square, the calculations lead to the following expression:

$$
\int_{0}^{\infty} d t[f(t)]^{2}=\pi \int_{0}^{\infty} d t\left[\frac{\operatorname{erf}(t)}{t}\right]^{2}+2 \sqrt{\pi} \int_{0}^{\infty} \frac{d t}{t^{3}} \operatorname{erf}(t)\left(e^{-t^{2}}-1\right)+\int_{0}^{\infty} \frac{d t}{t^{4}}\left(e^{-t^{2}}-1\right)^{2}
$$

All integrals appearing in the right-hand-side of Eq.(8) can be calculated exactly in analytic form. The final results are listed below:

$$
\begin{gathered}
\int_{0}^{\infty} d t\left[\frac{\operatorname{erf}(t)}{t}\right]^{2}=\frac{4}{\sqrt{\pi}} \sinh ^{-1}(1) \\
\int_{0}^{\infty} \frac{d t}{t^{3}} \operatorname{erf}(t)\left(e^{-t^{2}}-1\right)=1-\sqrt{2}-\sinh ^{-1}(1),
\end{gathered}
$$

and

$$
\int_{0}^{\infty} \frac{d t}{t^{4}}\left(e^{-t^{2}}-1\right)^{2}=\frac{4 \sqrt{\pi}}{3}(\sqrt{2}-1)
$$

In the above expressions, $\sinh ^{-1}(x)=\ln \left(x+\sqrt{x^{2}+1}\right)$ is the inverse hyperbolic sine function. After some careful algebra, one eventually ends up with:

$$
\int_{0}^{\infty} d t[f(t)]^{2}=2 \sqrt{\pi}\left[\sinh ^{-1}(1)+\frac{1-\sqrt{2}}{3}\right],
$$

where $\sinh ^{-1}(1)=\ln (1+\sqrt{2})$. Details of the calculations of the integrals in Eq.(9), Eq.(10) and Eq.(11) can be found, respectively, in Appendix. A, Appendix. B, and Appendix. C.

The exact calculation of the Coulomb self-energy of a uniformly charged cube [21], which involves a sixdimensional integral, is immediate by using the same approach:

$$
\frac{U}{k \rho_{0}^{2} L^{5}}=\frac{1}{\sqrt{\pi}} \int_{0}^{\infty} d t f(t)^{3} .
$$

For a $3 \mathrm{D}$ uniform charge distribution over the volume of a cube we have:

$$
k \rho_{0}^{2} L^{5}=\frac{k Q^{2}}{L} \quad(3 D),
$$

since $\rho_{0}=Q / L^{3}$ in such a case. The calculation of the integral $\int_{0}^{\infty} d t[f(t)]^{3}$ in Eq.(13) follows a similar path as that of $\int_{0}^{\infty} d t[f(t)]^{2}$. However, the hint at this point is that the calculations are neither trivial, nor ordinary. Therefore, for the sake of brevity, we limit ourselves to state that the integrals resulting from the cubic expansion of $\left[f_{1}(t)+f_{2}(t)\right]^{3}$ can all be calculated exactly by carefully handling the apparent singularities of individual integrals at the origin. Without further elaboration, the final result for this case is shown below:

$\frac{U}{k \rho_{0}^{2} L^{5}}=\frac{1+\sqrt{2}-2 \sqrt{3}}{5}-\frac{\pi}{3}+\ln [(1+\sqrt{2})(2+\sqrt{3})]$.

One notes that the function $f(t)$ in Eq.(7) can be easily handled numerically since it is well-behaved in the $t \rightarrow 0$ and $t \rightarrow \infty$ limits. Although we believe that straightfoward careful integration may be the only way to calculate analytically integrals involving arbitrary powers of $f(t)$, other possibilities cannot be excluded. For instance, the following two properties:

$$
\begin{aligned}
& \frac{d}{d t} f(t)=-\frac{f(t)}{t}+\frac{1-e^{-t^{2}}}{t^{3}} \\
& \int f(t) d t=2 t_{2} F_{2}\left(\frac{1}{2}, \frac{1}{2} ; \frac{3}{2}, \frac{3}{2} ;-t^{2}\right)-t f(t),
\end{aligned}
$$

where ${ }_{2} F_{2}$ is a generalized hypergeometric function may prove to be of some use for recurrence methods.

\section{COULOMB SELF-ENERGY INTEGRAL OF A UNIFORMLY CHARGED D-CUBE}

Calculation of the Coulomb self-energy integral of a uniform charge distribution in a $2 \mathrm{D}$ or $3 \mathrm{D}$ domain is a challenging task and often is done numerically. For instance, a very precise computational method aimed at the numerical evaluation of the six-dimensional integrals arising from Coulomb interactions in $3 \mathrm{D}$ is reported in Ref. [22]. In that case, techniques borrowed from integral geometry were used to reduce the number of integration variables from six to two. 
However, we showed in Section II that the expression for the Coulomb self-energy of a uniformly charged 2D square or $3 \mathrm{D}$ cube always reduces to the calculation of an integral with a single variable. In both approaches, the analytic singularities due to Coulomb's law are eliminated. Therefore, the reduction of the general problem to a single integration greatly benefits from the approach started in [20, 21]. Based on these considerations, we are now in a position to solve the general problem of a uniformly charged $d$-cube with a Coulomb self-energy given by the expression:

$\frac{U}{k \rho_{0}^{2} L^{2 d-1}}=\frac{1}{2} \prod_{i=1}^{d} \int_{0}^{1} d x_{i} \prod_{i=1}^{d} \int_{0}^{1} d x_{i}^{\prime}\left(\sum_{i=1}^{d}\left(x_{i}-x_{i}^{\prime}\right)^{2}\right)^{-\frac{1}{2}}$

Regardless of any computational technique, it is obvious that the problem is best solved by reducing it to a single integral. By using the same approach as that in 2D and 3D one obtains the following result:

$$
\frac{U}{k \rho_{0}^{2} L^{2 d-1}}=\frac{1}{\sqrt{\pi}} \int_{0}^{\infty} d t f(t)^{d}
$$

Note that $k \rho_{0}^{2} L^{2 d-1}=k Q^{2} / L$ is the appropriate unit of energy for a $d$-cube since in such a case, $\rho_{0}=Q / L^{d}$. It is quite possible that one can solve analytically the integral appearing in the right-hand-side of Eq.(18) for any $d \geq 4$. However, any analytic calculation is expected to be very lengthy and challenging even for the simplest case of $d=$ 4. On the other hand, the numerical calculation of the $1 \mathrm{D}$ integral expression in Eq.(18) is very easy and can be carried out very precisily for any value of $d$. We computed a large number of Coulomb self-energy integrals for values of up to $d=10^{6}$. In Table I we give the values of the integral, $\frac{1}{\sqrt{\pi}} \int_{0}^{\infty} d t f(t)^{d}$ (Coulomb self-energy in units of $\left.k Q^{2} / L\right)$ for all integer values of $d$ starting from $d=2$ up to $d=28$.

\begin{tabular}{|c||c|c|c||c|c|}
\hline$d$ & Value & $d$ & Value & $d$ & Value \\
\hline 2 & 1.48660 & 11 & 0.38990 & 20 & 0.28167 \\
3 & 0.94115 & 12 & 0.37141 & 21 & 0.27448 \\
4 & 0.74071 & 13 & 0.35531 & 22 & 0.26783 \\
5 & 0.63084 & 14 & 0.34117 & 23 & 0.26163 \\
6 & 0.55920 & 15 & 0.32861 & 24 & 0.25585 \\
7 & 0.50775 & 16 & 0.31734 & 25 & 0.25044 \\
8 & 0.46847 & 17 & 0.30716 & 26 & 0.24535 \\
9 & 0.43718 & 18 & 0.29791 & 27 & 0.24057 \\
10 & 0.41149 & 19 & 0.28944 & 28 & 0.23605 \\
\hline
\end{tabular}

TABLE I. Numerically computed values of $\frac{1}{\sqrt{\pi}} \int_{0}^{\infty} d t f(t)^{d}$ for $d=2,3, \ldots, 28$. The values diminish monotonically as the dimension $d$ of the hypercube increases. See text for details.

We now explore how the Coulomb self-energy of a $d$ cube varies as a function of the dimension of space. A first look of the results seemed to suggest a single powerlaw dependence as a function of $d$. However, upon further scrutiny, it was noted that this was not the case. We found out that there exists a sum of different contributions that follow a well-defined power-law pattern.

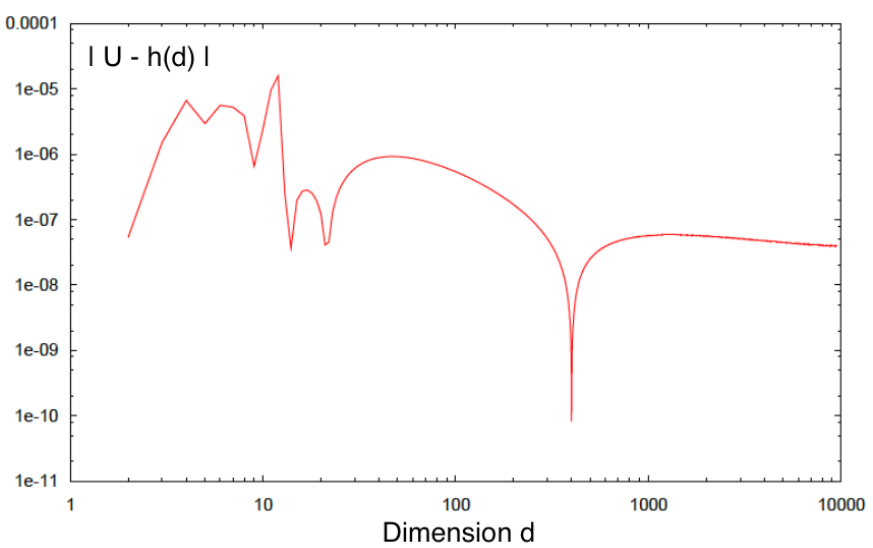

FIG. 1. (color online). Absolute difference between $U$ (in units of $\left.k Q^{2} / L\right)$ and the fitting function in Eq.(19) as a function of the dimensionality $d$ of the uniformly charged hypercube. Notice the extraordinary agreement of the results. See text for details.

We carried out a fit of the Coulomb self-energy values $U$ (in units of $k Q^{2} / L$ ) as a function of the $d$-cube dimensionality for $d$ up to 10000 . The results shed light on the functional form of Eq.(18) which can be accurately approximated by the following fitting function:

$$
h(d)=\sum_{i=1}^{\infty} a_{i} d^{-\frac{2 i-1}{2}}
$$

The most relevant contribution to the fitting function, $h(d)$ comes only from the first six terms of the series with coefficients: $a_{i}=$ $\{1.22474,0.644393,0.94151,3.03579,-4.52733,7.15345\}$, all of them with an error less than $0.01 \%$. This function is a trans-series, a non-usual (non-Taylor) series expansion of a function. As a matter of fact, $h(d)$ is classified as a Hahn series. In mathematics, Hahn series are a type of formal infinite series. The algebraic theory of generalized power series is well developed and can be found in the literature under the name Hahn series. They are a generalization of Puiseux series (themselves a generalization of Taylor power series), first introduced by H. Hahn [23]. The excellent approximation of the result in Eq.(18) provided by $h(d)$ can be seen in Fig. 1. Despite the success of the function $h(d)$ to describe the Coulomb self-energy of a hypercube in $d$-dimensions, we cannot say, at this stage, whether the sum can be considered as a kind of perturbation series or an exact expansion. 


\section{DISCRETE-CHARGE APPROACH TO THE SELF-ENERGY INTEGRAL WITH CORRECTION DUE TO FINITE SIZE EFFECTS}

So far, we have seen that the Coulomb self-energy integral of a uniformly charged $d$-cube can be expressed as an infinite series of different power-law contributions (though, the quantity can be approximated very accurately by only a small number of terms). We will now investigate what happens when the dimensionality of the hypercube is fixed to a given value. We recall that the Coulomb self-energy integral is taken over a continuum where a total charge $Q$ is uniformly spread over a $d$ cube domain. This results in a uniform charge density $\rho_{0}=Q / L^{d}$. Earlier, we pointed out that the exact calculation of the Coulomb self-energy of a uniformly charged $d$-cube is extremely challenging for an arbitrary $d$. Thus, this model represents an excellent case study to gauge the accuracy and feasibility of various numerical schemes.

To this effect, we want to introduce a numerical approach that is meant to be both simple and sufficiently accurate in order to be useful. The basic idea behind the scheme is to approximate the continuous uniform charge distribution with a finite discrete distribution of $N$ point charges in a lattice. The discrete system should reflect the properties of the uniform charge distribution that is trying to approximate, namely, same total charge and same overall volume/geometry. Assuming that the continuous uniform charge distribution has a total charge, $Q$, let us consider a system of $N$ identical point charges each carrying the amount of charge $q$ so that the total charge of the discrete system is:

$$
N q=Q
$$

The point charges are placed in a regular lattice (this way the discrete system is a good representation of the uniformly charged continuous distribution). The nature of the lattice chosen for the array of point charges (cubic, hexagonal, etc.) is not that important. The final result is insensitive to the choice of the lattice as long as the number of point charges that occupy the lattice sites is sufficiently large. For example, in 2D, one can fill all space with squares or hexagons. Thus, one can set up either a 2D square or a 2D hexagonal/triangular lattice if so desires. The same applies to any given dimensionality. If $n$ is the number of point charges along the length, $L$ of the hypercube, then the separation distance between charges is given by $\Delta L=L /(n-1)$. The total number of point charges is $N=n^{d}$. Placing more charges inside the hypercube with fixed length is tantamount of saying that $\Delta L$ diminishes, and, thus, the discrete system comes closer to the continuum. Fig. 2 illustrates the situation for a cubic lattice of unit length containing a total of $N=20^{3}=8000$ point charges.

We denote as $U_{N}$ the total Coulomb electrostatic energy corresponding to the discrete system of $N$ identical point charges, $q$ placed in a hypercube with length, $L$

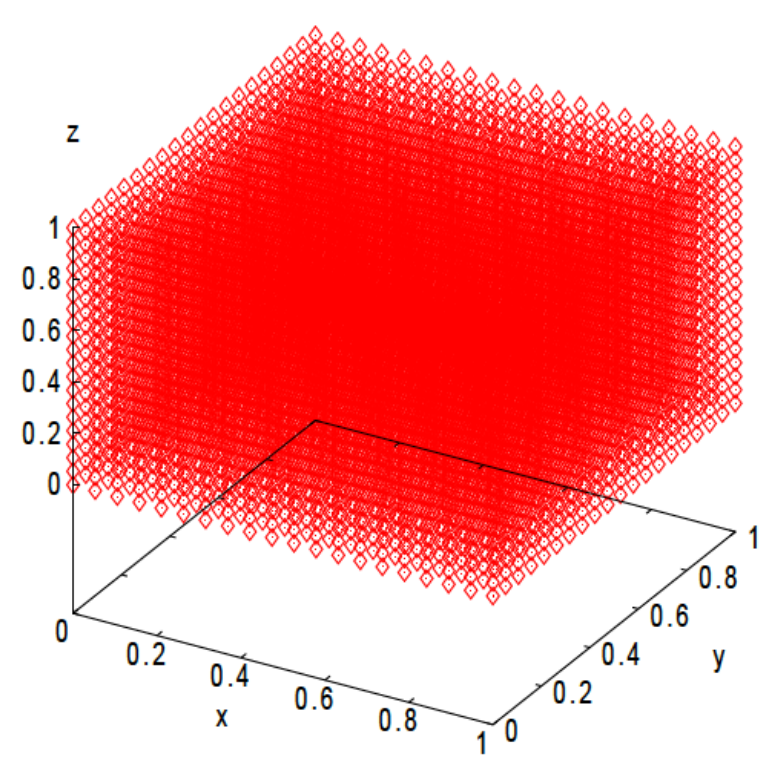

FIG. 2. (color online). A cube of unit length containing $N=20^{3}=8000$ point charges placed in a cubic lattice. The larger the number of point charges, the closer we come to the continuum limit. See text for details.

and arbitrary dimensionality, $d$. This term is written as:

$$
U_{N}=\sum_{i<j}^{N} \frac{k q^{2}}{\left|\vec{r}_{i}-\vec{r}_{j}\right|},
$$

where $\left|\vec{r}_{i}-\vec{r}_{j}\right|$ should be interpreted as a separation distance between two point charges in $d$-space. Since $q=Q / N$, one can write Eq.(21) as:

$$
U_{N}=\frac{k Q^{2}}{L} \frac{1}{N^{2}} \sum_{i<j}^{N} \frac{1}{\left|\vec{r}_{i}-\vec{r}_{j}\right| / L} .
$$

The energy $U_{N}$ of the discrete system serves as a zeroth order approximation to the exact self-energy $U$ of the continuous charge distribution. One easily recognizes that the quantity $\frac{1}{N^{2}} \sum_{i<j}^{N} \frac{1}{\left|\vec{r}_{i}-\vec{r}_{j}\right| / L}$ represents the discrete counterpart to the exact continuous value, $U /\left(k Q^{2} / L\right)=U /\left(k \rho_{0}^{2} L^{2 d-1}\right)$. This means that the exact self-energy of the $d$-cube (in units of $k Q^{2} / L$ ) has the quantity $I_{N} / N^{2}$ as its discrete system counterpart where

$$
I_{N}=\sum_{i<j}^{N} \frac{1}{r_{i j}} .
$$

In the above expression, $r_{i j}=\left|\vec{r}_{i}-\vec{r}_{j}\right| / L$ represents a dimensionless separation distance between charges in $d$-space. Since there are $N(N-1) / 2$ contributions to the sum above, we expect the leading term in $I_{N}$ to be roughly of $O\left(N^{2}\right)$. Although our calculations have the $d$-hypercube in mind, we point out that the results obtained should not depend on the particular topology of 
hyperspace and hence should be valid for any distribution of charges in $\mathbb{R}^{d}$. Obviously, it is expected that, for small $N$, the quantity $I_{N} / N^{2}$ may not be a good approximation to $U$ (in units of $k Q^{2} / L$ ). Therefore, $I_{N} / N^{2}$ represents only a decent start, namely, a reasonable zero-th order approximation in a discretization approach to the calculation of a continuous integral. With this in mind, we can quite generally assume that a better approximation to the continuous self-energy integral could be written as:

$$
\frac{U}{k \rho_{0}^{2} L^{2 d-1}}=\frac{I_{N}}{N^{2}}+g(N)
$$

where $g(N)$ represents an additional first-order correction and the only correction term that we will incorporate. Such a correction would improve the accuracy of the discrete-to-continuum approximation. If the Coulomb self-energy of a given uniform charge distribution is known (as in the $d$-cube case), a numerical calculation of $I_{N} / N^{2}$ for selected values of $N$ would enable us to understand better the function $g(N)$. By the same token, knowledge of the functional form of $g(N)$, would enable us to infer the value of $U$ more accurately. Knowledge of $g(N)$ leads one to a better estimate of the value of $U$ by relying on calculations of discrete systems with a finite number of point charges. Simple considerations suggest that $g(N)$ should be proportional to the discretization step, $\Delta L=L /(n-1)$. In dimensionless units, $\Delta L / L=1 /(n-1)$ where $n=N^{\frac{1}{d}}$. Based on this reasoning, we expect $g(N)$ to go as $O\left(N^{-1 / d}\right)$.

We apply the following numerical scheme for the finite$N$ fit of the results at any given number, $N$ of point charges. Take $N_{1}, N_{2} \gg 1$ with $N_{1}>N_{2}$. Assume that $g(N)=c \cdot G(N)$, where $G(N)$ is the functional form of the first-order correction term and $c$ is a real number. In the limit of large $N$, we have:

$$
\frac{I_{N_{1}}}{N_{1}^{2}}+c \cdot G\left(N_{1}\right) \approx \frac{I_{N_{2}}}{N_{2}^{2}}+c \cdot G\left(N_{2}\right) .
$$

We can use Eq.(25) to obtain the value of $c$. Hence, we can estimate the value of the continuous self-energy from the discrete system with $N_{1}$ point charges as:

$$
\frac{I_{N_{1}}}{N_{1}^{2}}+\frac{\frac{I_{N_{1}}}{N_{1}^{2}}-\frac{I_{N_{2}}}{N_{2}^{2}}}{G\left(N_{2}\right)-G\left(N_{1}\right)} G\left(N_{1}\right),
$$

that allows us to obtain any desirable precision. Obviously, in this scheme, there is no need to make a fit in order to find $c$ every time we estimate the quantity under consideration.

At this juncture, we remark on the importance of the results by pointing out that the method employed is universal and applies to general situations. This means that one can use the outlined method for any given uniformly charged body with an arbitrary shape irrespective of the nature of the interaction potential. The value of the selfenergy integral obtained from the finite discrete system will not depend on the choice of the lattice in the $N \rightarrow \infty$ limit. For all practical purposes, the value obtained numerically will be very accurate as long as the number of point charges that occupy the sites of the chosen lattice inside the body under consideration is large. Obviously, the different arrangement of point charges for a given lattice (cubic, hexagonal, etc.) may lead to a different termination of the lattice at the surface boundary of the body. This will result on values of self-energy that will slightly depend on the choice of the lattice since the number $N$ of point charges used in a real calculation is always finite. The treatment considered is also straightforward and easy to implement since the computation of self-energy integrals does not involve any computational complexity to tackle multiple integration.

\section{A. First-order correction of the finite energy in one dimension}

1D Coulomb chains consisting of up to several tens of ions have recently been created in experiments. Interparticle separations, on the order of micrometers, are sufficiently large so that a classical description is valid for most experiments. This fact validates a classical approach to the problem. The $1 \mathrm{D}$ chain has been suggested as an advantageous configuration for a novel type of atomic clock as well as for quantum computer schemes (see Ref.[24] and references therein). However, the selfenergy of a uniformly charged 1D body diverges if a standard Coulomb (inverse distance) interaction potential is used to describe the interaction between charges in 1D. Therefore, if we want to calculate the electrostatic selfenergy of a uniformly charged 1D rod, we must choose an appropriate interaction potential (of logarithmic form) for such a case:

$$
U=\frac{k \rho_{0}^{2}}{2} \int_{0}^{L} d x \int_{0}^{L} d x^{\prime} V\left(\left|x-x^{\prime}\right|\right)
$$

where $V\left(\left|x-x^{\prime}\right|\right)=-\ln \left|x-x^{\prime}\right|$ and $\rho_{0}=Q / L$. The exact result can be written as: $2 U /\left(k \rho_{0}^{2}\right)=L^{2}(3 / 2-$ $\ln L)$. Note that the potential $-\ln \left|x-x^{\prime}\right|$ cannot be rescaled. By using the fact that $\rho_{0}=Q / L$, one writes

$$
U=k Q^{2} \frac{1}{2}\left(\frac{3}{2}-\ln L\right) \text {. }
$$

As we did earlier, let us now consider a discrete system of $N$ identical point charges, $q$ placed along the length, $L$ of the rod at equal spacing between each other. The total electrostatic energy that corresponds to this discrete 1D system is written as:

$$
U_{N}=\sum_{i<j}^{N} k q^{2} V\left(\left|x_{i}-x_{j}\right|\right) .
$$

Since $q=Q / N$, one can write Eq.(29) as:

$$
U_{N}=k Q^{2} \frac{1}{N^{2}} \sum_{i<j}^{N} V\left(\left|x_{i}-x_{j}\right|\right) .
$$


The energy $U_{N}$ of the discrete system serves as an approximation to the exact self-energy $U$ of the continuous 1D uniform charge distribution. Thus, one easily recognizes that the counterpart to $U /\left(k Q^{2}\right)$ is $I_{N} / N^{2}$ where

$$
I_{N}=\sum_{i<j}^{N} V\left(\left|x_{i}-x_{j}\right|\right) \text {. }
$$

If the interaction potential between point charges in $1 \mathrm{D}$ would have been the usual Coulomb interaction potential we would have:

$$
\sum_{i<j}^{N} \frac{1}{\left|x_{j}-x_{i}\right|}=\sum_{i<j}^{N} \frac{1}{\Delta L(j-i)}
$$

where $\Delta L$ is the separation distance between two nearestneighboring point charges placed along a 1D array with total length, $L$. It is easy to prove that the quantity: $\sum_{i<j}^{N} \frac{1}{(j-i)}$ in the right-hand-side of Eq.(32) reduces to: $1+N[H(N-1)-1][7]$, where $H(p)=\sum_{n=1}^{p} \frac{1}{n}=$ $1+1 / 2+\ldots+1 / p$ is a finite harmonic series. Obviously, $H(N-1)=1+1 / 2+\ldots+1 /(N-1)$ is the sum of the first $(N-1)$ terms of the harmonic series. The relation mentioned above can be easily proved by induction starting from $H(N-1)=\ln (N-1)+\gamma+O(1 / N)$, where $\gamma$ is the Euler constant. This leads to $(N-1)^{2}[\gamma-1+\ln (N-$ $1)+O(\ln N / N)]$. Thus, there is good reason to believe that the first-order correction added to $I_{N} / N^{2}$ (in 1D) may be of the order $O(\ln N / N)$ for $N$ sufficiently large. As we shall see, this is indeed the case. The energy difference $\left|I_{N} / N^{2}-U\right|$ as a function of $N$ is shown in Fig. 3 for $L=3$ where $I_{N}$ is calculated from Eq.(31) and $U$ is given in units of $k Q^{2}$.

The earlier arguments together with known results for planar charge distributions [7] suggest a good candidate for the first-order energy correction term (in 1D) in the form of the function, $g(N) \sim c \ln N / N$ where $c$ is a fitting constant. The inset of Fig. 3 shows a plot $\left(\left|I_{N} / N^{2}-U\right|\right) /(\ln N / N)$ as a function of $N$. Analysis of the results indicates that a good fit is achieved for $c \sim 10$. It is not strange to obtain a functional form of this kind if we recall the celebrated Wigner's crystal result (see, e.g., Ref. [24]). For a fixed density the energy per particle and the chemical potential diverge logarithmically as the number of particles $N$ increases, which is a consequence of the long-range nature of the Coulomb interaction potential. In a $2 \mathrm{D}$ or $3 \mathrm{D}$ system of electrons the problem is solved by the "jellium" model, which restores charge neutrality by adding a background of uniform charge of the opposite sign. However, 1D systems are peculiar since introduction of a uniform opposite charge would lead to a logarithmic divergence in the short-range part of the Coulomb interaction potential. At this stage, we can take advantage of the (now) known first-order energy correction term to provide a better estimate of the corresponding self-energy of uniformly charged continuous distribution. The upper curve in Fig. 4 represents the same as

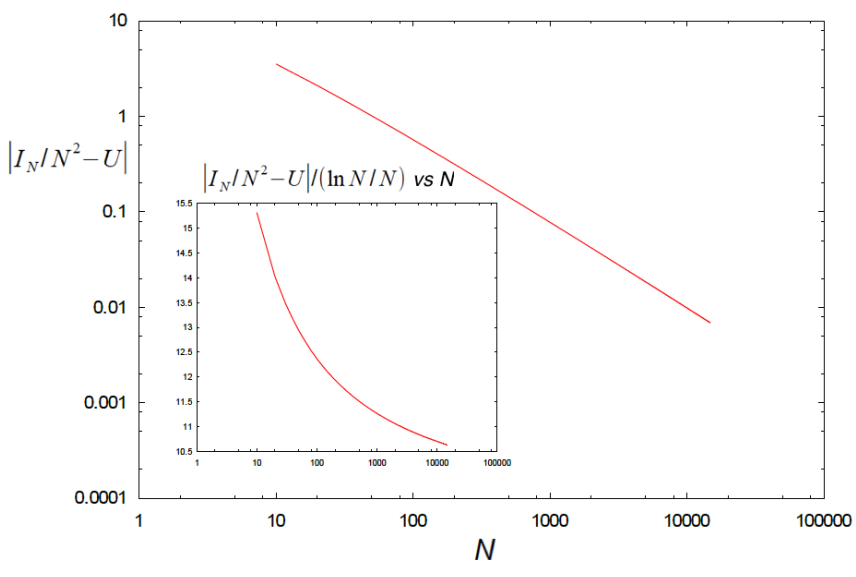

FIG. 3. (color online). Energy difference $\left|I_{N} / N^{2}-U\right|$ for the case of a uniformly charged $1 \mathrm{D}$ rod as a function of the number, $N$ of point charges in the finite system. The selfenergy $U$ of the uniformly charged rod is given in units of $k Q^{2}$. The first impression is that the curve manifests a powerlaw decay. However, upon closer inspection, it is shown that the quantity approaches a constant value times $\ln N / N$ (see inset) in the $N \rightarrow \infty$ limit. See text for details.

in Fig. 3. Note the spectacular accuracy boost achieved when the first-order correction term, $g(N)=c \ln N / N$ is added to $I_{N} / N^{2}$. This means that we are able to compute involved integrals using a simple discrete sum in conjunction with a first-order correction term that considerably improves the accuracy of the estimate. In 1D, we obtain an accuracy of four digits for a relatively modest number of $N=10^{3}$ point charges.

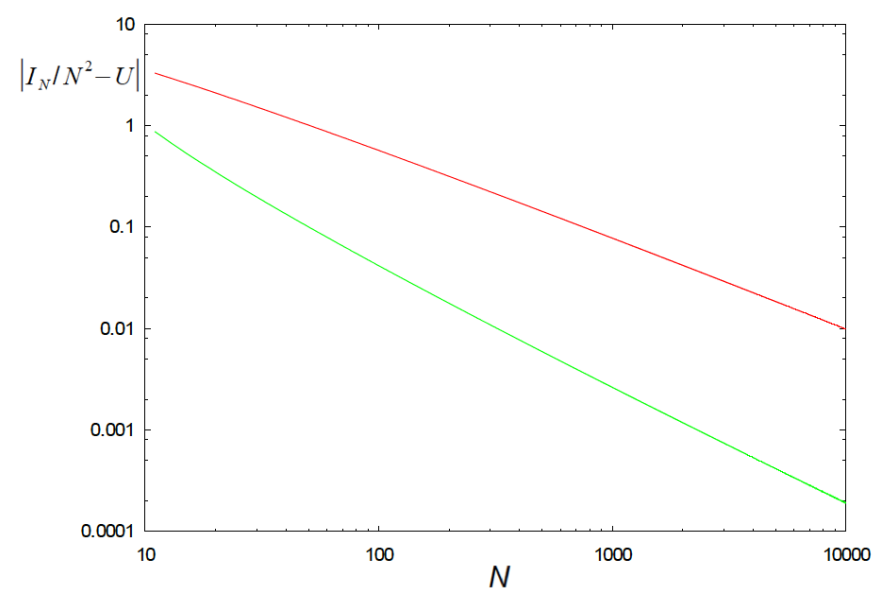

FIG. 4. (color online). Same plot as in Fig. 3 (upper curve). The addition of the first-order finite-size correction term considerably improves the approximation (lower curve). See text for details. 


\section{B. First-order correction of the finite energy in two dimensions}

The case of 2D Coulomb self-energy integrals is certainly of great relevance, for in the relation: $\frac{U}{k Q^{2} / L}=$ $\frac{I_{N}}{N^{2}}+g(N)$, the first-order correction term, $g(N)$ is expected to go as $c / \sqrt{N}$. This sort of dependence of $g(N)$ on $N$ is exactly the same as the probabilistic statistical "error" of Monte Carlo methods. This coincidence validates the efficiency of the method for computing the selfenergy integrals for any potential by using the discretization approach introduced earlier. The absolute difference

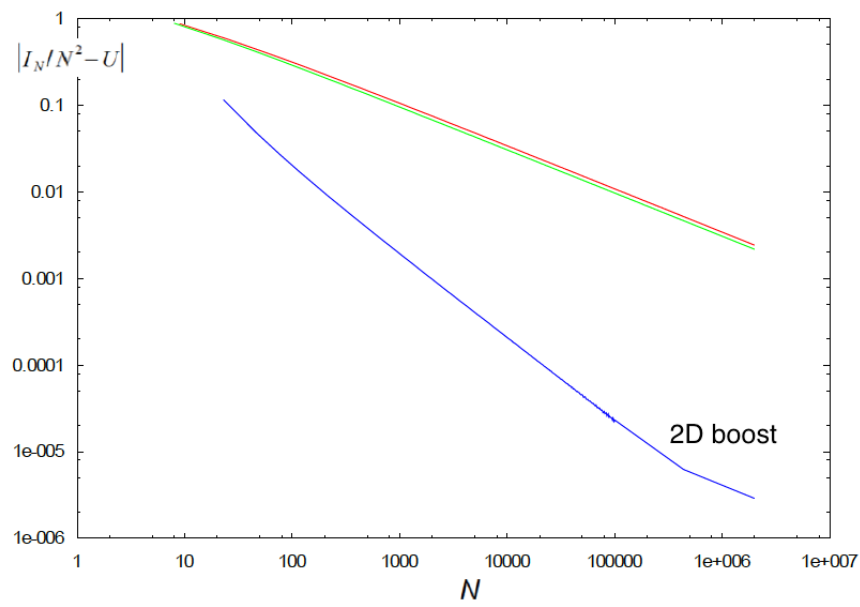

FIG. 5. (color online). Energy difference $\left|I_{N} / N^{2}-U\right|$ for a uniformly charged $2 \mathrm{D}$ square as a function of the total number of point charges $N$ used in the finite discrete sum. The Coulomb self-energy, $U$ is given in units of $k Q^{2} / L$. The upper curve uses a square grid to cover the domain, while the lower curve employes a hexagonal grid. Although slightly better, a hexagonal grid does not provide a considerable advantage. Note the spectacular accuracy of the results when the firstorder energy correction term is included. See text for details.

between the approximated value from the discrete system and the exact value of the Coulomb self-energy integral of a uniformly charged $2 \mathrm{D}$ square, $\left|I_{N} / N^{2}-U /\left(k Q^{2} / L\right)\right|$ is shown in Fig. 5. The results for the upper curve are obtained by placing the point charges in a square lattice, while the slightly lower curve reflects the same quantity but for a hexagonal lattice. The finite hexagonal grid manifests a slightly better accuracy since it allows for a denser packing of the area of the square. The error for $N=10^{6}$ point charges in a grid is of the same order of magnitude as that corresponding to the same number of trials using Monte Carlo integration techniques. However, one must appreciate the fact that we are using a deterministic approach. This means that, in theory, we are providing a better estimate to the exact value without any statistical uncertainty (that is inherent in a Monte Carlo method). This is clearly seen from Fig. 5, where the accuracy boost is spectacular when the first-order energy correction term is taken into account. It is, thus, obvious that the current deterministic method is far more precise than a traditional Monte Carlo calculation.

\section{First-order correction of the finite energy in three, four and five dimensions}

The $3 \mathrm{D}$ case shows same features as the $2 \mathrm{D}$ one. In Fig. 6 we plot the energy difference $\left|I_{N} / N^{2}-U /\left(k Q^{2} / L\right)\right|$ for the cases of a 3D, 4D and 5D uniformly charged hypercube (upper curves). One notes a significant improvement of the accuracy in the 3D case when the first-order correction, $g(N)=O\left(N^{-1 / 3}\right)$ is added. Again, the computational boost provided by the first-order correction term is sizeable and considerably improves the accuracy of the approximation. Analysis of the results for $d$-cubes

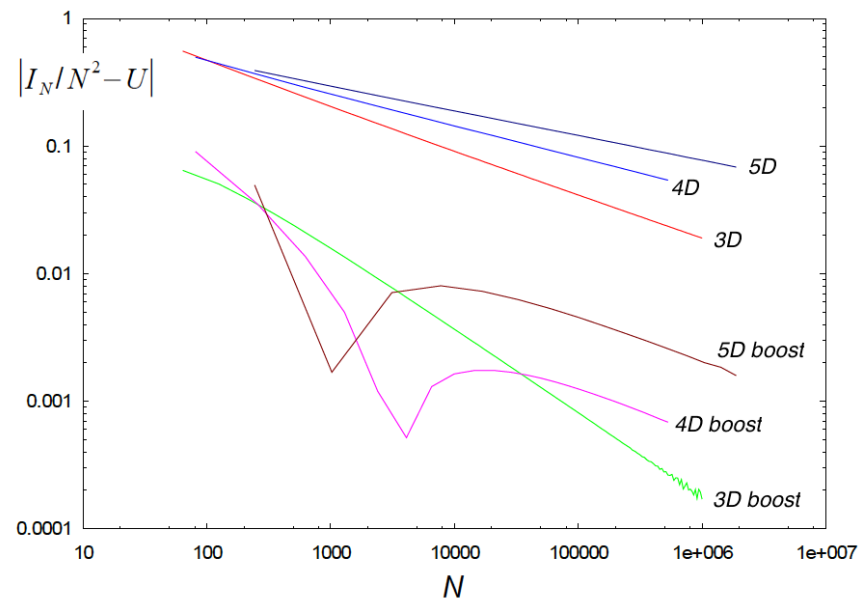

FIG. 6. (color online). Energy difference $\left|I_{N} / N^{2}-U\right|$ for a uniformly charged three-, four- and five-dimensional cube as a function of the total number of point charges $N$ used in the finite sum. The Coulomb self-energy, $U$ is given in units of $k Q^{2} / L$. It is apparent that, for the same $N$, we have less accuracy in the results due to the corrections given by $c / N^{1 / d}$. See text for details.

with larger dimensionalities, $d=4$ and 5 leads to similar conclusions. Note that a larger number of point charges in the grid is required in order to obtain the same accuracy in $d=4$ and 5 as that in $d=3$ since the firstorder corrections are now of the order $O\left(N^{-1 / 4}\right)$ and $O\left(N^{-1 / 5}\right)$, respectively. For the most challenging case of $d=5$, the inferred result provides only three exact digits of accuracy while estimating the corresponding ten-fold integral continuum integral. Overall, this is quite acceptable if one takes into account the simplicity of the approach when compared to the numerical cost of developing sophisticated numerical algorithms to deal with the same problem. 


\section{CONCLUSIONS}

In this work we employed a very useful mathematical transformation that applies to a standard Coulomb interaction potential $\left(\propto 1 /\left|\vec{r}-\vec{r}^{\prime}\right|\right)$ between point charges to calculate the resulting Coulomb self-energy of a uniformly charged $d$-cube with an arbitrary dimensionality. The method allows one to obtain a general expression for the Coulomb self-energy of a uniformly charged $d$-cube as a simple integral of an auxiliary function that depends on a single variable. The resulting integral can be calculated numerically with very high precision for any dimensionality $d(\geq 2)$. The results obtained allow one to investigate the dependence of the Coulomb self-energy of a uniformly charged multi-dimensional cube as a function of its dimensionality, $d$. It was found that the Coulomb self-energy of a uniformly charged $d$-cube $d(\geq 2)$ can be fitted very accurately by a trans-series of power functions that employes only a few terms. The unique form of the trans-series deserves attention and is quite useful since it allows one to study the problem analytically.

Since the exact calculation of the Coulomb self-energy of a uniformly charged $d$-cube is extremely challenging, we considered it as a case study to gauge the accuracy of a very simple numerical approach that can be used to calculate the self-energy of arbitrarily-shaped uniform charge distributions. In this scheme, a continuous uniform charge distribution is modeled as a discrete finite system of $N$ point charges that are placed in a given finite regular lattice so that the total charge of the discrete system and the volume occupied by the discrete system are the same as that of its continuum counterpart. The energy contribution from the finite system of interacting point charges in a grid together with a first-order energy correction is found to be a very accurate representation of the Coulomb self-energy of uniformly charged $d$-cubes. It was found that the first-order energy correction in 2D works exactly as the statistical error induced by Monte Carlo integration techniques. The good accuracy obtained for the case of uniformly charged $d$-cubes leads us to believe that this method can be applied succesfully to other uniformly charged domains with a more complex geometry such as convex bodies, regions with holes or simply irregular domains. The results derived can be used in systematic studies of the properties of finite systems of charges in different geometries and domains. One of the great advantages of the current approach for approximating continuum integrals, is its simplicity. No highly-involved numerical techniques are used and results are obtained with a very reasonable accuracy. In addition, the method is easy to generalize and applies to any given potential (like the logarithmic potential in 1D).

\section{ACKNOWLEDGEMENTS}

J. Batle acknowledges fruitful discussions with J. Rosselló, Maria del Mar Batle and Regina Batle. The research of one of the authors (O. C.) was supported in part by US Army Research Office (ARO) grant no. W911NF13-1-0139 and National Science Foundation (NSF) grant no. DMR-1410350. M. N. and M. G. acknowledge support from Kermanshah Branch, Islamic Azad University, Kermanshah, Iran.

\section{Appendix A: Calculation of the integral $\int_{0}^{\infty} d x\left[\frac{\operatorname{erf}(x)}{x}\right]^{2}$}

We consider the integral:

$$
\int_{0}^{\infty} d x\left[\frac{\operatorname{erf}(x)}{x}\right]^{2}
$$

where $\operatorname{erf}(x)$ is an error function. We use integration by parts $\left(\int u d v=u v-\int v d u\right)$ where $u=[\operatorname{erf}(x)]^{2}$ and $d v=d x / x^{2}$. It is straightforward to obtain:

$$
\int_{0}^{\infty} d x\left[\frac{\operatorname{erf}(x)}{x}\right]^{2}=\frac{4}{\sqrt{\pi}} \int_{0}^{\infty} d x e^{-x^{2}} \frac{\operatorname{erf}(x)}{x} .
$$

The integral appearing in the right-hand-side of Eq.(A2) can be calculated from the following formula:

$$
\int_{0}^{\infty} d x e^{-a^{2} x^{2}} \frac{\operatorname{erf}(x)}{x}=\sinh ^{-1}\left(\frac{1}{\sqrt{a^{2}}}\right),
$$

where $\sinh ^{-1}(x)=\ln \left(x+\sqrt{x^{2}+1}\right)$ is the inverse hyperbolic sine function. By using the result from Eq.(A3), one has:

$$
\int_{0}^{\infty} d x\left[\frac{\operatorname{erf}(x)}{x}\right]^{2}=\frac{4}{\sqrt{\pi}} \sinh ^{-1}(1) .
$$

Additional details about this calculation are found in Ref. [25].

\section{Appendix B: Calculation of the integral

$$
\int_{0}^{\infty} \frac{d x}{x^{3}} \operatorname{erf}(x)\left(e^{-x^{2}}-1\right)
$$

We consider the integral:

$$
I=\int_{0}^{\infty} \frac{d x}{x^{3}} \operatorname{erf}(x)\left(e^{-x^{2}}-1\right),
$$

where $\operatorname{erf}(x)$ is an error function. We write it as:

$$
I=I_{1}-I_{2},
$$


where

$$
I_{1}=\int_{0}^{\infty} \frac{d x}{x^{3}} e^{-x^{2}} \operatorname{erf}(x)
$$

and

$$
I_{2}=\int_{0}^{\infty} \frac{d x}{x^{3}} \operatorname{erf}(x)
$$

When treated separately, both $I_{1}$ and $I_{2}$ have singularities. However, we will be prove that such singularities cancel out when the difference, $I_{1}-I_{2}$ is calculated. We start the calculation of $I_{1}$ by using integration by parts $\left(\int u d v=u v-\int v d u\right)$ where $u=e^{-x^{2}} \operatorname{erf}(x)$ and $d v=d x / x^{3}$. We obtain:

$$
I_{1}=-\left.\frac{1}{2} \frac{e^{-x^{2}} \operatorname{erf}(x)}{x^{2}}\right|_{0} ^{\infty}-\int_{0}^{\infty} \frac{d x}{x} e^{-x^{2}} \operatorname{erf}(x)+\frac{1}{\sqrt{\pi}} \int_{0}^{\infty} \frac{d x}{x^{2}} e^{-2 x^{2}}
$$

The first term in the right-hand-side of Eq.(B5) is divergent for $x=0$ since one can see that:

$$
\lim _{x \rightarrow 0} \frac{e^{-x^{2}} \operatorname{erf}(x)}{x^{2}}=\infty
$$

In order to keep track of this singular terms, we write Eq.(B5) as:

$$
I_{1}=\left.\frac{1}{2} \frac{e^{-x^{2}} \operatorname{erf}(x)}{x^{2}}\right|_{x \rightarrow 0}-\int_{0}^{\infty} \frac{d x}{x} e^{-x^{2}} \operatorname{erf}(x)+\frac{1}{\sqrt{\pi}} \int_{0}^{\infty} \frac{d x}{x^{2}} e^{-2 x^{2}} .
$$

The first integral in the right-hand-side of Eq.(B7) has no singularities:

$$
\int_{0}^{\infty} \frac{d x}{x} e^{-x^{2}} \operatorname{erf}(x)=\sinh ^{-1}(1)
$$

where $\sinh ^{-1}(x)=\ln \left(x+\sqrt{x^{2}+1}\right)$ is the inverse hyperbolic sine function. However, the second integral in the right-hand-side of Eq.(B7) has singularities. We start its calculation by first considering the following formula for an indeterminate integral:

$$
\int \frac{d x}{x^{2}} e^{-a^{2} x^{2}}=-\frac{e^{-a^{2} x^{2}}}{x}-\sqrt{\pi} \operatorname{aerf}(a x) .
$$

By using the formula in Eq.(B9) one writes the corresponding determinate integral (with lower boundary, $x=0$ and upper boundary, $x=\infty)$ as:

$$
\int_{0}^{\infty} \frac{d x}{x^{2}} e^{-a^{2} x^{2}}=\left.\frac{e^{-a^{2} x^{2}}}{x}\right|_{x \rightarrow 0}-\sqrt{\pi a^{2}}
$$

Note that: $\operatorname{aerf}(a x)=|a| \operatorname{erf}(|a| x)$ where $|a|=\sqrt{a^{2}}$. Based on Eq.(B10), one concludes that:

$$
\int_{0}^{\infty} \frac{d x}{x^{2}} e^{-2 x^{2}}=\left.\frac{e^{-2 x^{2}}}{x}\right|_{x \rightarrow 0}-\sqrt{2 \pi} .
$$

The results from Eq.(B8) and $\underset{\mathrm{Eq}}{\mathrm{Eq}}$.(B11) are then substituted into Eq.(B7) to obtain:

$$
I_{1}=\left.\frac{1}{2} \frac{e^{-x^{2}} \operatorname{erf}(x)}{x^{2}}\right|_{x \rightarrow 0}-\sinh ^{-1}(1)+\left.\frac{1}{\sqrt{\pi}} \frac{e^{-2 x^{2}}}{x}\right|_{x \rightarrow 0}-\sqrt{2} .
$$

One uses a similar approach to calculate $I_{2}$ from
Eq.(B4) by first considering the indeterminate integral:

$$
\int \frac{d x}{x^{3}} \operatorname{erf}(x)=-\frac{1}{\sqrt{\pi}} \frac{e^{-x^{2}}}{x}-\operatorname{erf}(x)-\frac{\operatorname{erf}(x)}{2 x^{2}} .
$$


By applying the formula obtained from Eq.(B13) to the corresponding determinate integral (with lower boundary, $x=0$ and upper boundary, $x=\infty$ ) in conjunction with a careful consideration of the appropriate $x \rightarrow 0$ and $x \rightarrow \infty$ limits, one concludes that:

$$
I_{2}=-1+\left.\frac{1}{\sqrt{\pi}} \frac{e^{-x^{2}}}{x}\right|_{x \rightarrow 0}+\left.\frac{\operatorname{erf}(x)}{2 x^{2}}\right|_{x \rightarrow 0} .
$$

By substituting the results from Eq.(B12) and Eq.(B14) to the expression in Eq.(B2), one can easily verify that all singular terms in the $x \rightarrow 0$ limit cancel out exactly when one calculates, $I=I_{1}-I_{2}$. The final result reads:

$$
I=1-\sqrt{2}-\sinh ^{-1}(1) .
$$

\section{Appendix C: Calculation of the integral $\int_{0}^{\infty} \frac{d x}{x^{4}}\left(e^{-x^{2}}-1\right)^{2}$}

This integral can be written as:

$$
\int_{0}^{\infty} \frac{d x}{x^{4}}\left(e^{-x^{2}}-1\right)^{2}=\int_{0}^{\infty} \frac{d x}{x^{4}} e^{-2 x^{2}}-2 \int_{0}^{\infty} \frac{d x}{x^{4}} e^{-x^{2}}+\int_{0}^{\infty} \frac{d x}{x^{4}}
$$

Each of the integrals in the right-hand-side of Eq.(C1) can be calculated by using the following formula:

$$
\begin{aligned}
\int \frac{d x}{x^{4}} e^{-a^{2} x^{2}}= & e^{-a^{2} x^{2}}\left(-\frac{1}{3 x^{3}}+\frac{2 a^{2}}{3 x}\right) \\
& +\frac{2}{3} a^{3} \sqrt{\pi} \operatorname{erf}(a x) .
\end{aligned}
$$

We recall that:

$$
\lim _{x \rightarrow 0} \operatorname{erf}(a x)=0 \quad ; \quad \lim _{x \rightarrow \infty} \operatorname{erf}(a x)= \pm 1=\frac{a}{|a|} .
$$

As a result the value of the determinate integral in Eq.(C2) (with lower boundary, $x=0$ and upper bound- ary, $x=\infty$ ) reads:

$$
\begin{aligned}
\int_{0}^{\infty} \frac{d x}{x^{4}} e^{-a^{2} x^{2}}= & \left.e^{-a^{2} x^{2}}\left(\frac{1}{3 x^{3}}-\frac{2 a^{2}}{3 x}\right)\right|_{x \rightarrow 0} \\
& +\frac{2}{3} \sqrt{\pi}\left(a^{2}\right)^{3 / 2} .
\end{aligned}
$$

We wrote $|a|^{3}=\left(a^{2}\right)^{3 / 2}$ in order to keep the result in Eq.(C4) as general as possible. Note that each of the integrals appearing in the right-hand-side of Eq.(C1) contains a divergent term in the $x \rightarrow 0$ limit. However, one can verify that all such divergent terms cancel out exactly when added up in the right-hand-side expression of Eq.(C1). The final result becomes:

$$
\int_{0}^{\infty} \frac{d x}{x^{4}}\left(e^{-x^{2}}-1\right)^{2}=\frac{4}{3} \sqrt{\pi}(\sqrt{2}-1)
$$

[1] E. P. Wigner, Phys. Rev. B 46, 1002 (1934).

[2] C. C. Grimes and G. Adams, Phys. Rev. Lett. 42, 795 (1979).

[3] E. Y. Andrei, G. Deville, D. C. Glattli, F. I. B. Williams, E. Paris, and B. Etienne, Phys. Rev. Lett. 60, 2765 (1988).

[4] V. V. Deshpande and M. Bockrath, Nat. Phys. 4, 314 (2008).

[5] O. Ciftja, NANO 10, No. 8, 1550114 (2015).

[6] J. J. Thomson, Phil. Mag. 7, 237 (1904).

[7] J. Batle, A. Bagdasaryan, M. Abdel-Aty, and S. Abdalla, Physica A 451, 237 (2016).

[8] A. Mughal and M. A. Moore, Phys. Rev. 76, 011606 (2007).
[9] D. P. Hardin and E. B. Saff, Not. Am. Math. Soc. 51, 1186 (2004).

[10] Y. Levin and J. J. Arenzon, Europhys. Lett. 63, 415 (2003).

[11] A. Mughal, Forma 29, 13 (2014).

[12] Yu. E. Lozovik and V. A. Mandelshtam, Phys. Lett. A 165, 469 (1992).

[13] F. Bolton and U. Rössler, Superlatt. Microstruct. 13, 139 (1992).

[14] V. Bedanov and F. M. Peeters, Phys. Rev. B 49, 2667 (1994).

[15] M. Kong, B. Partoens, A. Matulis, and F. M. Peeters, Phys. Rev. E 69, 036412 (2004).

[16] O. Ciftja, Physica B 458, 92 (2015). 
[17] R. G. Parr and W. Yang, Density-Functional Theory of Atoms and Molecules (Oxford Univ. Press, New York, 1989).

[18] J. M. Seminario (Ed.), Recent Developments and Applications of Modern Density Functional Theory (Elsevier, Amsterdam, 1996).

[19] D. J. Adams and G. S. Dubey, J. Comput. Phys. 27, 156 (1987).

[20] O. Ciftja, Phys. Lett. A 374, 981 (2010).
[21] O. Ciftja, Phys. Lett. A 375, 766 (2011).

[22] D. Finocchiaro, M. Pellegrini, and P. Bientinesi, J. Comput. Phys. 146, 707 (1998).

[23] H. Hahn, Über die nicht-archimedischen Größensysteme, reprinted in: Collected works Vol. 1, Springer-Verlag 1995.

[24] D. H. E. Dubin, Phys. Rev. E 55, 4017 (1997).

[25] O. Ciftja, J. Electrostat. 71, 102 (2013). 